\title{
Regorafenib plus modified FOLFOX6 as first-line treatment of metastatic colorectal cancer: A phase II trial is
}

\author{
Guillem Argilés ${ }^{\mathrm{a}}$, Mark P. Saunders ${ }^{\mathrm{b}}$, Fernando Rivera ${ }^{\mathrm{c}}$, Alberto Sobrero ${ }^{\mathrm{d}}$, \\ Al Benson III ${ }^{\mathrm{e}}$, Carmen Guillén Ponce ${ }^{\mathrm{f}}$, Stefano Cascinu ${ }^{\mathrm{g}}$, Eric Van Cutsem ${ }^{\mathrm{h}}$, \\ Iain R. Macpherson ${ }^{i}$, Dirk Strumberg ${ }^{j}$, Claus-Henning Köhne ${ }^{k}$, John Zalcberg ${ }^{1}$, \\ Andrea Wagner ${ }^{\mathrm{m}}$, Vittorio Luigi Garosi ${ }^{\mathrm{n}}$, Julia Grunert ${ }^{\mathrm{o}}$, Josep Tabernero ${ }^{\mathrm{a}}$, \\ Fortunato Ciardiello ${ }^{\mathrm{p}, *}$
}

\author{
a Vall d'Hebron University Hospital and Institute of Oncology (VHIO), Universitat Autònoma de Barcelona, P. Vall d'Hebron 119-129, \\ 08035 Barcelona, Spain \\ ${ }^{\mathrm{b}}$ The Christie, 550 Wilmslow Road, Manchester M20 4BX, UK \\ ${ }^{\text {c } U n i v e r s i t y ~ H o s p i t a l ~ ' M a r q u e ́ s ~ d e ~ V a l d e c i l l a ', ~ A v d a . ~ D e ~ V a l d e c i l l a ~ 25, ~} 39008$ Santander Cantabria, Spain \\ ${ }^{\mathrm{d}}$ IRCCS San Martino IST Hospital, Largo Rosanna Benzi 10, Genova 16132, Italy \\ ${ }^{\mathrm{e}}$ Robert H. Lurie Comprehensive Cancer Center, Northwestern University, 676 N. St. Clair, Suite 850, Chicago, IL 60611, USA \\ ${ }^{\mathrm{f}}$ University Hospital Ramón y Cajal, Carretera de Colmenar Viejo, km. 9,100, 28034 Madrid, Spain \\ ${ }^{\mathrm{g}}$ University Hospital Riuniti Umberto I, Via Conca, 60126 Ancona, Italy \\ ${ }^{\mathrm{h}}$ University Hospitals and KU Leuven, Herestraat 49, 3000 Leuven, Belgium \\ ${ }^{\mathrm{i}}$ Beatson West of Scotland Cancer Centre, 1053 Great Western Road, Glasgow G12 0YN, UK \\ ${ }^{\mathrm{j}}$ University Hospitals, Marienhospital Herne, Hölkeskampring 40, 44625 Herne, Germany \\ ${ }^{\mathrm{k}}$ Oldenburg Clinic, Rahel-Straus-Straße 10, 26133 Oldenburg, Germany \\ ${ }^{1}$ School of Public Health and Preventive Medicine Faculty of Medicine, Nursing and Health Sciences, Monash University, The Alfred Centre, 99 \\ Commercial Rd, Melbourne 3004, Australia \\ ${ }^{\mathrm{m}}$ Bayer Pharma AG, Müllerstraße 178, 13353 Berlin, Germany \\ ${ }^{\mathrm{n}}$ Bayer S.p.A., Viale Certosa 210, 20156 Milan, Italy \\ ${ }^{\circ}$ Bayer Pharma AG, Aprather Weg 18, 42096 Wuppertal, Germany \\ ${ }^{\mathrm{p}}$ Second University Hospital, Via S Pansini 5, 80131 Naples, Italy
}

Received 29 October 2014; received in revised form 9 February 2015; accepted 19 February 2015 Available online 25 March 2015

\footnotetext{
Initial results from this trial were presented at the American Society of Clinical Oncology Annual Meeting, Chicago, IL, USA, 31 May to 4 June 2013.

* Corresponding author at: Dipartimento di Internistica Clinica e Sperimentale 'F. Magrassi', Seconda Università degli studi di Napoli, Via S. Pansini 5, 80131 Naples, Italy. Tel.: +39081566 6745; fax: +39081566 6732.

E-mail addresses: gargiles@vhebron.net (G. Argilés), Mark.Saunders@christie.nhs.uk (M.P. Saunders), oncrhf@humv.es (F. Rivera), alberto. sobrero@hsanmartino.it (A. Sobrero), ABenson@nmff.org, a-benson@northwestern.edu (A. Benson III), carmenguillenponce@gmail.com (C. Guillén Ponce), cascinu@yahoo.com (S. Cascinu), Eric.VanCutsem@uzleuven.be (E. Van Cutsem), Iain.Macpherson@glasgow.ac.uk (I.R. Macpherson), dirk.strumberg@marienhospital-herne.de (D. Strumberg), koehne.claus-henning@klinikum-oldenburg.de (C.-H. Köhne), John.Zalcberg@petermac.org (J. Zalcberg), andrea.wagner2@bayer.com (A. Wagner), VittorioLuigi.Garosi@bayer.com (V. Luigi Garosi), julia. grunert@bayer.com (J. Grunert), fortunato.ciardiello@unina2.it (F. Ciardiello).
} 


\section{KEYWORDS}

Colorectal neoplasms

Fluorouracil

Leucovorin

Oxaliplatin

Regorafenib

Safety

Survival rate

Treatment outcome

\section{Introduction}

Every year, more than 1.36 million patients worldwide are diagnosed with colorectal cancer (CRC) and nearly 700,000 deaths are attributed to this disease [1]. At the time of diagnosis, up to $25 \%$ of patients present with metastatic disease, while $50-60 \%$ of patients with CRC will develop metastases at some point $[2,3]$. Standard treatment for these patients consists of a fluoropyrimidine-based chemotherapy backbone combined with other systemic cytotoxic agents, such as oxaliplatin and irinotecan, together with monoclonal antibodies such as bevacizumab and, in patients with $R A S$ wildtype tumours, cetuximab or panitumumab. These therapies have improved overall survival (OS) from six to around 20-24 months $[2,4]$.

Regorafenib is an oral multikinase inhibitor that blocks the activity of a variety of protein kinases involved in the regulation of oncogenesis (KIT, RET, RAF1, BRAF and BRAF ${ }^{\mathrm{V} 600 \mathrm{E}}$ ), angiogenesis (vascular endothelial growth factor [VEGF] receptors $1-3$ and TIE2) and the tumour microenvironment (plateletderived growth factor receptor and fibroblast growth factor receptor) [5]. The phase III CORRECT trial demonstrated the efficacy and tolerability of regorafenib monotherapy in patients with previously treated metastatic CRC [6]. On the basis of those findings, regorafenib monotherapy has been approved internationally for patients with metastatic CRC previously treated with other available standard therapies.

A phase I study of regorafenib in combination with 5-fluorouracil (5-FU) + folinic acid with either oxaliplatin (FOLFOX) or irinotecan (FOLFIRI) demonstrated an acceptable tolerability profile in patients with metastatic CRC [7]. Pharmacokinetic data revealed increased exposure to irinotecan, as well as its active metabolite $\mathrm{SN}-38$, when administered in combination with regorafenib; however, regorafenib did not significantly affect the pharmacokinetics of either 5-FU or oxaliplatin [7]. The current study was designed to assess the activity and tolerability of regorafenib in combination with a modified FOLFOX regimen (mFOLFOX6) as first-line therapy for metastatic CRC.

\section{Materials and methods}

\subsection{Study design and participants}

The CORDIAL trial (ClinicalTrials.gov identifier: NCT01289821) was an international, multicentre, single-arm, open-label, phase II exploratory study conducted at 16 centres in Australia, Belgium, Spain, Germany, Italy, the United Kingdom (UK) and the United States of America (USA) (investigators at each site are listed in the Supplementary appendix). Ethical approval of the study protocol was provided by each centre's institutional review board or independent ethics committee. The trial followed the principles of the Declaration of Helsinki and good clinical practice, complying with all local laws and regulations. All patients provided written informed consent before enrolment.

Patients had to be at least 18 years old, with histological or cytological documentation of 
adenocarcinoma of the colon or rectum, and had to be suitable to receive first-line treatment with mFOLFOX6 for metastatic disease. Patients had to have at least one measurable lesion according to Response Evaluation Criteria in Solid Tumors (RECIST) version 1.1 [8], an Eastern Cooperative Oncology Group performance status of 0 or 1, adequate bone-marrow, liver and renal function and an anticipated life expectancy of at least 3 months. Exclusion criteria included previous systemic anticancer therapy for metastatic CRC (although adjuvant chemotherapy for stages I-III CRC was permitted provided that therapy had ceased $>6$ months before screening and disease recurrence was documented), previous treatment with anti-VEGF therapies or signal transduction inhibitors or uncontrolled hypertension (systolic $>150 \mathrm{~mm} / \mathrm{Hg}$ or diastolic $>90 \mathrm{~mm} / \mathrm{Hg}$ ) despite optimal management.

Patient recruitment commenced in February 2011 and was stopped in July 2011 when the target number of patients was reached. The data cut-off date for the present analyses was 15th November 2012. The last end-of-survival follow-up visit took place in June 2014.

\subsection{Procedures}

Patients were treated with a combination of mFOLFOX6 plus regorafenib. The mFOLFOX6 regimen was administered according to normal clinical practice starting on days 1 and 15 of each 28-day cycle and consisted of oxaliplatin $85 \mathrm{mg} / \mathrm{m}^{2}$ and folinic acid $400 \mathrm{mg} / \mathrm{m}^{2}$, both as 2-h intravenous (IV) infusions, and 5 -FU $400 \mathrm{mg} / \mathrm{m}^{2}$ as an IV bolus immediately followed by a $2400 \mathrm{mg} / \mathrm{m}^{2}$ IV infusion over $46 \mathrm{~h}$. Regorafenib was given orally as a single morning dose of $160 \mathrm{mg}$ on days 4-10 and 18-24 of each 28-day cycle. The treatment schedule is summarised in Fig. 1.

Treatment continued until death, tumour progression (defined by RECIST), unacceptable toxicity, withdrawal of consent or investigator's decision to stop. If an individual drug had to be withdrawn because of toxicity, the patient could continue to receive the remaining components until one of the above criteria was met. Dose modifications were implemented for any individual component of the combination therapy to manage toxicities related to that drug. If patients stopped all components of mFOLFOX6 and continued on single-agent regorafenib, the treatment regimen for regorafenib was modified to $160 \mathrm{mg}$ once daily in repeating cycles of 3 weeks on/ 1 week off treatment according to the schedule used for regorafenib when given as monotherapy.

\subsection{Study end-points}

The primary end-point was objective response rate (ORR) based on blinded central radiological review. ORR was defined as the proportion of patients with a best overall tumour response of partial or complete response. Secondary end-points included disease control rate (DCR; sum of complete response + partial response + stable disease), progression-free survival (PFS), OS, duration of response and duration of stable disease. In addition, the safety and tolerability of the regimen were evaluated using adverse events and changes in laboratory measures (haematology, chemistry and urinalysis), vital signs (blood pressure, heart rate and temperature) and electrocardiogram. Although not a prespecified end-point, investigator-reported duration of treatment was also assessed.

\subsection{Assessments}

Tumour assessment by CT or MRI was performed at screening and every two cycles throughout the study until disease progression was documented, using identical techniques at each assessment. Unless consent to follow up was specifically withdrawn, survival assessments were made every 2 months until death, via review of medical records and regular contact. Safety was monitored continuously until $30 \pm 4$ days after discontinuation of treatment.

\subsection{Statistical analysis}

Statistical evaluation and estimation, using SAS version 9.1 or higher (SAS Institute Inc., Cary, NC, USA), were based on a one-sided type I error level of $10 \%$ and a two-sided confidence level of $80 \%$, respectively.

The primary end-point (ORR) was analysed based on a one-sample exact binomial test. The aim was to assess whether regorafenib plus mFOLFOX6 significantly

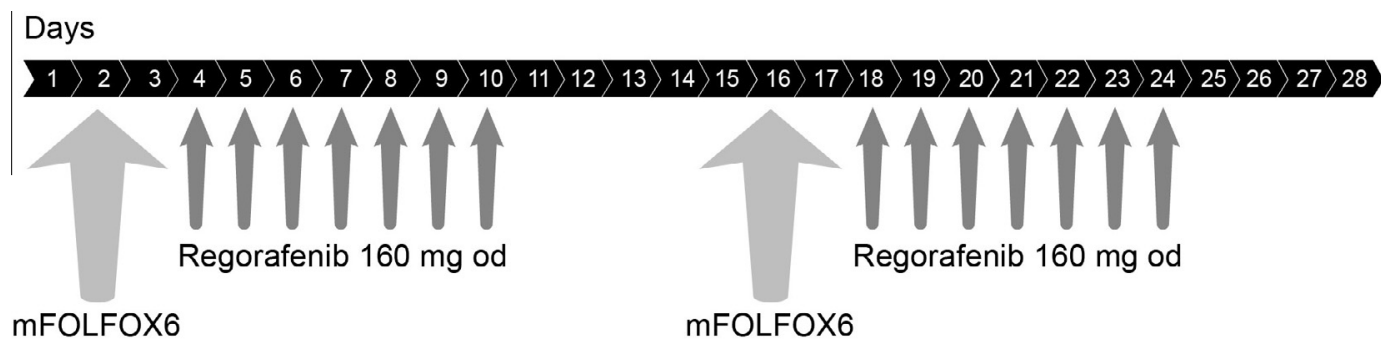

Fig. 1. Treatment schedule (28-day cycle). mFOLFOX6, modified regimen of folinic acid +5 -fluorouracil + oxaliplatin 6; od, once-daily. 


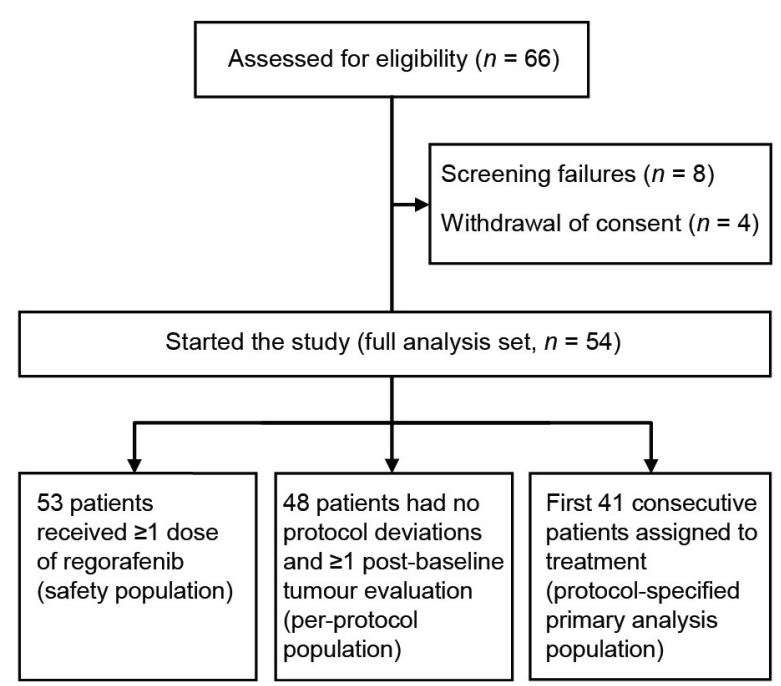

Fig. 2. Flow of patients through the study.

improved the ORR compared with that seen in a similar population of historical cohorts. With a total planned sample size of 41 evaluable patients in the primary analysis set (i.e. all patients who were evaluated for objective response), the null hypothesis was to be rejected if at least 21 patients $(51 \%)$ were classified as responders. The estimated response rate and its twosided $80 \%$ confidence interval $(\mathrm{CI})$ were given.

Variables measured on interval scales were summarised using descriptive statistics, while frequency tables were provided for variables measured on nominal scales. Time-to-event data were displayed using KaplanMeier estimates for survival functions.

\section{Results}

\subsection{Patient demographics and characteristics}

In total, 66 patients were screened for inclusion in CORDIAL; 54 patients started the study and were included in the full analysis set (FAS; Fig. 2). Patient demographic and baseline tumour characteristics are presented in Table 1.

At the time of data cut-off, five patients were still receiving regorafenib treatment (regorafenib $+5-\mathrm{FU}$, $n=2$; regorafenib +5 -FU infusion only, $n=2$; regorafenib monotherapy, $n=1$ ). In November 2013, one patient was still receiving regorafenib monotherapy. Treatment duration and reasons for discontinuations are summarised in Table 2.

\subsection{Efficacy}

In the primary analysis set $(n=41)$, a confirmed ORR was observed in $43.9 \%$ of patients ( $n=18$; all partial responses), while DCR (complete response + partial response + stable disease) was observed in $85.4 \%$ of
Table 1

Patient demographic and baseline tumour characteristics (full analysis set, $N=54$ ).

\begin{tabular}{llc}
\hline Characteristic & $n$ & $\%$ \\
\hline Male:female ratio & $28: 26$ & $51.9: 48.1$ \\
Age (years) & & \\
$\quad$ Median (range) & $61(32-80)$ & 61.1 \\
$\quad<65$ & 33 & 33.3 \\
$65-75$ & 18 & 5.6 \\
$\quad \geqslant 75$ & 3 & \\
ECOG performance status & & \\
0 & 35 & 64.8 \\
1 & 19 & 35.2 \\
Primary disease site & & \\
Colon & 35 & 64.8 \\
Rectum & 15 & 27.8 \\
Colon and rectum & 4 & 7.4 \\
TNM stage at diagnosis & & \\
I & 1 & 1.9 \\
IIa & 5 & 9.3 \\
IIIb/c & 7 & 13.0 \\
IV & 41 & 75.9 \\
Number of target lesions & & 3.7 \\
0 & 2 & 5.6 \\
1 & 3 & 13.0 \\
$2-3$ & 42 & \\
$4-5$ & 7 & \\
\hline
\end{tabular}

ECOG, Eastern Cooperative Oncology Group; TNM, tumour, node, metastasis.

Table 2

Treatment duration and reasons for discontinuation (full analysis set, $N=54)$.

\begin{tabular}{lll}
\hline & $\begin{array}{l}\text { Any study } \\
\text { treatment }^{\mathrm{a}}\end{array}$ & Regorafenib $^{\mathrm{b}}$ \\
\hline $\begin{array}{l}\text { Treatment duration } \\
\text { Months (median [range]) }\end{array}$ & $9.9(0.6-19.6)$ & $7.7(0.1-19.5)$ \\
$\begin{array}{l}\text { Cycles (median [range]) } \\
\text { Patients on treatment }\end{array}$ & $8(1-21)$ & $7(1-21)$ \\
$>6$ months (n [\%]) & $26(48.1)$ & $29(53.7)$ \\
$\begin{array}{l}\text { Patients on treatment }>1 \text { year } \\
(n[\%])^{\mathrm{c}}\end{array}$ & $6(11.1)$ & $6(11)$ \\
Reasons for treatment discontinuation $(n[\%])$ & \\
$\quad$ Disease progression & $39(72.2)$ & \\
Adverse events & $4(7.4)$ & \\
Investigator's decision & $3(5.6)$ & \\
Patient withdrawal & $1(1.9)$ & \\
\hline
\end{tabular}

${ }^{a}$ Regorafenib, oxaliplatin, folinic acid and/or 5-fluorouracil (5-FU).

${ }^{\mathrm{b}}$ As combination therapy or monotherapy.

${ }^{\mathrm{c}}$ Regorafenib + mFOLFOX6, $n=1$; regorafenib +5 -FU, $n=2$; regorafenib +5 -FU infusion only, $n=2$; regorafenib monotherapy, $n=1$.

patients $(n=35)$; no patients achieved a complete response (Table 3). The findings were similar in the per-protocol analysis $(n=48$; see Table 3$)$.

Kaplan-Meier estimates of OS and PFS in the FAS are presented in Fig. 3. The median duration of clinical 
Table 3

Centrally assessed response using RECIST version 1.1.

\begin{tabular}{|c|c|c|c|c|c|}
\hline & & \multicolumn{2}{|c|}{ Primary analysis set $(N=41)$} & \multicolumn{2}{|c|}{ Per-protocol set $(N=48)$} \\
\hline & & $n(\%)$ & $80 \% \mathrm{CI}$ & $n(\%)$ & $80 \% \mathrm{CI}$ \\
\hline \multirow[t]{5}{*}{ Best overall response } & Complete response & 0 & $0.0-3.9$ & 0 & $0.0-3.3$ \\
\hline & Partial response & $18(43.9)$ & $33.2-55.1$ & $20(41.7)$ & $31.9-52.0$ \\
\hline & Stable disease & $17(41.5)$ & $30.9-52.7$ & $22(45.8)$ & $35.9-56.1$ \\
\hline & Progressive disease & $5(12.2)$ & $6.1-21.5$ & $5(10.4)$ & $5.2-18.5$ \\
\hline & Missing & $1(2.4)$ & $0.3-9.2$ & $1(2.1)$ & $0.22-7.9$ \\
\hline $\mathrm{DCR}^{\mathrm{a}}$ & & $35(85.4)$ & $75.7-92.1$ & $42(87.5)$ & $79.1-93.3$ \\
\hline $\mathrm{ORR}^{\mathrm{b}}$ & & $18(43.9)$ & $33.2-55.1$ & $20(41.7)$ & $31.9-52.0$ \\
\hline
\end{tabular}

CI, confidence interval; DCR, disease control rate; ORR, objective response rate; RECIST, Response Evaluation Criteria in Solid Tumors.

${ }^{a}$ DCR defined as complete response + partial response + stable disease (stable disease had to be maintained for $\geqslant 7$ weeks).

${ }^{\mathrm{b}}$ ORR defined as complete response + partial response.

A

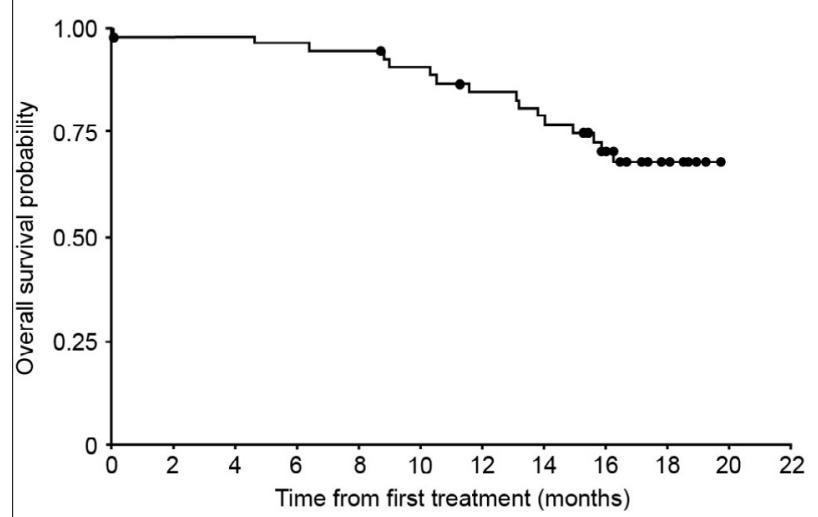

B

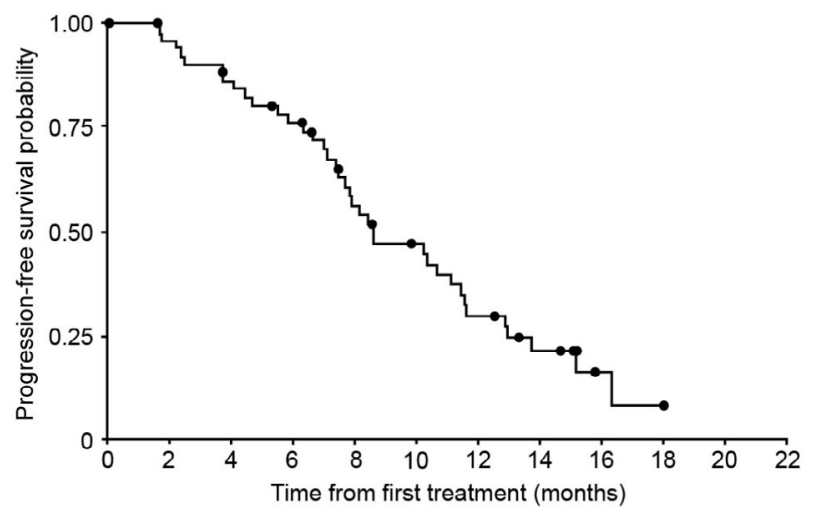

Fig. 3. Kaplan-Meier estimates of (A) overall survival (median not reached because $>50 \%$ of patients were still alive at the cut-off date; 38 censored [70.4\%]) and (B) progression-free survival (median 8.5 months, $95 \%$ confidence interval $7.4-11.3$ months; median time to treatment failure 7.8 months, $95 \%$ confidence interval 6.2 11.2 months). Full analysis set, $N=54$.

response in patients with an objective response $(n=20)$ was 9.0 months $(95 \%$ CI 6.6 months to not reached), while the median duration of stable disease in patients with stable disease as the best response $(n=22)$ was 7.6 months ( $95 \%$ CI $5.5-8.5$ months). Change in target lesion volume from baseline is shown in Fig. 4.

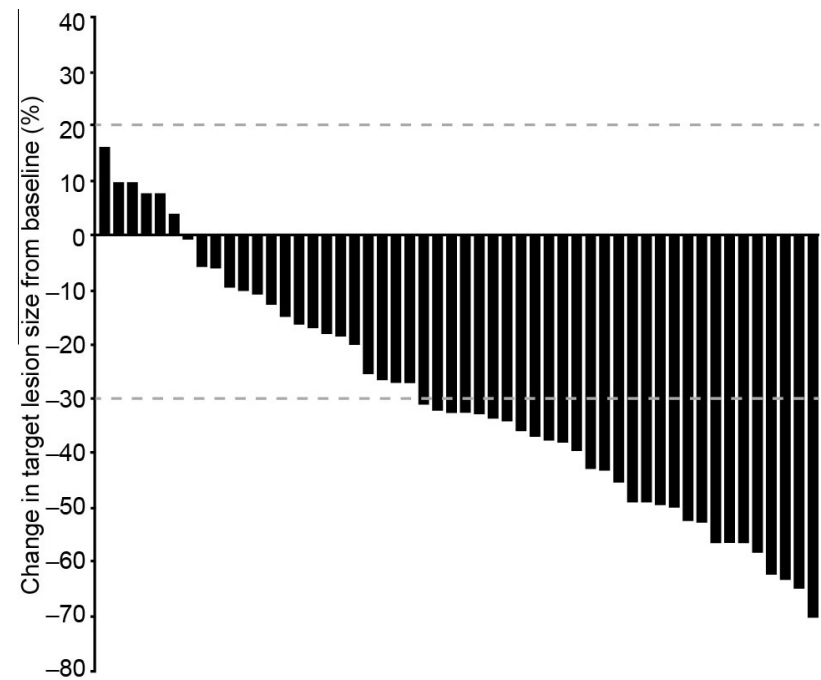

Fig. 4. Best change in target lesion size from baseline (full analysis set, $N=54$ ). Best change could not be determined in two patients. Six patients $(11.1 \%)$ had increases in tumour size, while 46 patients $(85.2 \%)$ had tumour shrinkage. Maximum tumour reductions of $\geqslant 30 \%$ were recorded in 29 patients $(53.7 \%)$.

\subsection{Safety}

In total, 52 patients $(98.1 \%)$ received greater than $90 \%$ of the planned regorafenib dose, with 51 patients $(96.2 \%)$ requiring regorafenib dose modifications (reductions or interruptions) as a result of adverse events (Table 4).

All 53 patients who received study medication experienced at least one treatment-emergent adverse event (TEAE) during the study. TEAEs affecting more than $20 \%$ of patients are presented in Table 5 . No grade 5 TEAEs were reported. Serious TEAEs occurred in 21 patients $(39.6 \%)$, with drug-related serious TEAEs reported in 13 patients $(24.5 \%)$. Overall, TEAEs led to dose modifications in 51 patients $(96.2 \%)$, with discontinuation of a component of study treatment required by 19 patients $(35.8 \%)$ and discontinuation of full study treatment in four patients $(8 \%)$. The most frequent 
Table 4

Dose modifications (safety population, $N=53$ ).

\begin{tabular}{ccccc}
\hline & Regorafenib & Oxaliplatin & 5-FU bolus & 5-FU infusion \\
\hline Patients requiring dose & interruptions or delays $(n[\%])$ \\
Any & $50(94.3)$ & $48(90.6)$ & $46(86.8)$ & $47(88.7)$ \\
$1-3$ & $27(50.9)$ & $21(39.6)$ & $19(35.8)$ & $18(33.9)$ \\
$>3$ & $23(43.4)$ & $27(50.9)$ & $27(50.9)$ & $29(54.7)$ \\
Patients requiring dose reductions $(n[\%])$ & \\
Any & $25(47.2)$ & $37(69.8)$ & $29(54.7)$ & $30(56.6)$ \\
1 & $15(28.3)$ & $25(47.2)$ & $28(52.8)$ & $22(41.5)$ \\
2 & $10(18.9)$ & $11(20.8)$ & $3(5.7)$ & $9(17.0)$ \\
\hline
\end{tabular}

5-FU, 5-fluorouracil.

adverse events leading to regorafenib dose reductions were diarrhoea and hand-foot skin reaction. The most frequent adverse events leading to oxaliplatin dose reduction were neurotoxicity, paraesthesia and diarrhoea, while the most frequent adverse events resulting in 5-FU dose reduction were neutropenia, diarrhoea and decreased platelets.

\section{Discussion}

The CORDIAL study failed to reach its primary endpoint, with the ORR in patients receiving regorafenib and mFOLFOX6 showing little difference from that seen with standard therapy alone. The combination of regorafenib and mFOLFOX6 was associated with a comparable median PFS ( 8.5 months) to FOLFOX and FOLFIRI chemotherapy alone ( 8.0 and 8.5 months, respectively) [9]. Median PFS in the current study appeared to be longer than that in most trials investigating chemotherapy and kinase inhibitor combinations (Supplementary Table 1) [10,11].
The apparent lack of improvement in tumour shrinkage following the addition of regorafenib to mFOLFOX6 versus historical data for chemotherapy alone (ORR of $43.9 \%$ versus an expected $35-55 \%$ for mFOLFOX6 alone $[9,12-16])$ together with the slowing of tumour growth observed in CORDIAL (as suggested by the higher than expected PFS) are consistent with results from the phase III CORRECT trial [6]. In CORRECT, regorafenib monotherapy significantly increased OS and PFS compared with placebo, despite a negligible ORR of $1.4 \%$ in a refractory population that had received multiple prior lines of therapy. The observations from CORDIAL and CORRECT could support a cytostatic rather than a cytotoxic effect of regorafenib on metastatic CRC. It may be possible to determine whether this theorised effect translates into prolonged OS once data are available from longer-term follow up of patients in CORDIAL.

The limitations of the CORDIAL study are its singlearm design and relatively small population. Despite the small sample size and exploratory nature of the trial, the PFS and ORR results are consistent with those reported with regimens containing other small-molecule kinase inhibitors (such as cediranib, vatalanib and sunitinib), while the duration of treatment in CORDIAL appears to be prolonged compared with those agents (Supplementary Table 1) [10,11,17].

The design of the CORDIAL study allowed modification of any component of the study treatment to manage adverse events. As a result of this tailored approach, the study showed that this combination regimen had a manageable tolerability profile in the first-line treatment of patients with metastatic CRC. Overall, the adverse event profile of the regorafenib plus mFOLFOX6

Table 5

Treatment-emergent adverse events occurring in $\geqslant 20 \%$ of patients (safety population, $N=53$ ).

\begin{tabular}{|c|c|c|c|c|c|}
\hline Adverse event ( $n[\%])$ & Any grade & Grade 1 & Grade 2 & Grade 3 & Grade 4 \\
\hline Diarrhoea & $37(69.8)$ & $16(30.2)$ & $9(17.0)$ & $12(22.6)$ & 0 \\
\hline Neutropenia & 34 (64.2) & $2(3.8)$ & $11(20.8)$ & $13(24.5)$ & $8(15.1)$ \\
\hline Fatigue & $34(64.2)$ & $20(37.7)$ & $12(22.6)$ & $2(3.8)$ & 0 \\
\hline Hypertension & $29(54.7)$ & $4(7.4)$ & $10(18.9)$ & $13(24.5)$ & $2(3.8)$ \\
\hline Paraesthesia & $28(52.8)$ & $14(26.4)$ & $9(17.0)$ & $5(9.4)$ & 0 \\
\hline Abdominal pain & $27(50.9)$ & $14(26.4)$ & $11(20.8)$ & $2(3.8)$ & 0 \\
\hline Nausea & $26(49.1)$ & $23(43.4)$ & $2(3.8)$ & $1(1.9)$ & 0 \\
\hline Thrombocytopenia & $25(47.2)$ & $10(18.9)$ & $11(20.8)$ & $4(7.5)$ & 0 \\
\hline Oral mucositis & $24(45.3)$ & $15(28.3)$ & $8(15.1)$ & $1(1.9)$ & 0 \\
\hline Peripheral neuropathy & $24(45.3)$ & $13(24.5)$ & $4(7.5)$ & $7(13.2)$ & 0 \\
\hline Anorexia & $19(35.8)$ & $15(28.3)$ & $3(5.7)$ & $1(1.9)$ & 0 \\
\hline Hand-foot skin reaction & $19(35.8)$ & $7(13.2)$ & $10(18.9)$ & $2(3.8)$ & 0 \\
\hline Constipation & $16(30.2)$ & $13(24.5)$ & $2(3.8)$ & $1(1.9)$ & 0 \\
\hline Dysgeusia & $16(30.2)$ & $14(26.4)$ & $2(3.8)$ & 0 & 0 \\
\hline Vomiting & $16(30.2)$ & $12(22.6)$ & $2(3.8)$ & $2(3.8)$ & 0 \\
\hline Alopecia & $15(28.3)$ & $13(24.5)$ & $2(3.8)$ & 0 & 0 \\
\hline Increased lipase & $14(26.4)$ & $4(7.5)$ & 0 & $9(17.0)$ & $1(1.9)$ \\
\hline Anaemia & $12(22.6)$ & $4(7.5)$ & $6(11.3)$ & $2(3.8)$ & 0 \\
\hline Voice alteration & $12(22.6)$ & $11(20.8)$ & $1(1.9)$ & 0 & 0 \\
\hline Increased aspartate aminotransferase & $12(22.6)$ & $8(15.1)$ & $1(1.9)$ & $3(5.7)$ & 0 \\
\hline Hypophosphataemia & $11(20.8)$ & 0 & $4(7.5)$ & $7(13.2)$ & 0 \\
\hline
\end{tabular}


combination appeared to be generally consistent with that of chemotherapy alone, although some regorafenib-related TEAEs, such as hand-foot skin reaction, were reported in addition to chemotherapy-related TEAEs. Of note, discontinuation of all study treatment (chemotherapy and regorafenib) as a result of TEAEs only occurred in four patients, with most patients continuing at least one component of study treatment [9,12-16].

In other studies of tyrosine kinase inhibitors in combination with chemotherapy, a decreased dose intensity of the chemotherapy components has been implicated in the apparent lack of efficacy. In CORDIAL, the intermittent dosing schedule could possibly underlie both the tolerability profile of regorafenib in combination with chemotherapy and the apparent antitumour activity, by avoiding any negative interaction between the impact of regorafenib on cell replication and the efficacy of the chemotherapy components. This hypothesis was first proposed in a serial imaging study of the VEGF receptor inhibitor axitinib; that study showed marked inhibition of fluorothymidine uptake during continuous treatment for 7 days, suggesting inhibition of cancer cell proliferation, which might protect against the cytotoxic effects of chemotherapy. The authors of that study hypothesised that this effect could explain the failure of VEGF receptor inhibitors in combination with chemotherapy regimens to improve outcomes [18].

Although duration of treatment was not a prespecified end-point, it is interesting to note that six patients received at least one component of study medication for 1 year or longer, with an overall median treatment duration for any component of study treatment of 9.9 months and five patients still receiving regorafenib therapy more than 6 months after data cut-off (regorafe$\mathrm{nib}+5$-FU, $n=2$; regorafenib +5 -FU infusion only, $n=2$; regorafenib monotherapy, $n=1$ ). The relatively long duration of treatment observed, albeit from a phase II study, contrasts with data from phase III studies of other first-line therapies for metastatic CRC, in which the duration of treatment rarely exceeds 6 months (Supplementary Table 2) [9,12-16].

The study did not meet its primary end-point, with no increase in the response rate compared with historical data from patients treated with mFOLFOX6 alone. Thus, the combination of regorafenib and mFOLFOX6 given in this schedule cannot be considered to be synergistic. However, given the median duration of treatment (9.9 months), together with some patients receiving at least one component of study therapy for more than 1 year, it would be interesting to explore the hypothesis that the addition of regorafenib to standard treatment might help patients to continue on treatment, and thus maintain tumour control for longer than might be achieved with chemotherapy alone.
Characterisation of patients who could derive the most long-term benefit, and the impact of dose modifications on tolerability and treatment duration, might provide the groundwork for investigation of the use of regorafenib as maintenance therapy in patients with metastatic $\mathrm{CRC}$ who have achieved a clinical response to cytotoxic chemotherapy.

\section{Author contributions}

J.T., F.C., A.B. III, C.-H.K., J.Z., A.W., V.L.G. and J.G. designed the trial, developed the protocol, coordinated the study and were responsible for management, analysis and interpretation of the data. G.A., M.P.S., F.R., A.S., A.B. III, C.G.-P., S.C., E.V.C., I.R.M., D.S., C.-H.K. J.Z., J.T. and F.C. enrolled patients. G.A., J.T., and F.C. were responsible for drafting the manuscript, with writing support from Succinct Medical Communications, funded by Bayer. All authors were responsible for review and revision of the manuscript, and approval of the submitted version.

\section{Conflict of interest statement}

Guillem Argilés: Bayer consultant for the development of regorafenib; Merck Serono consultant.

Mark P. Saunders: Bayer consultant.

Fernando Rivera: Research funding from Bayer, Amgen, Roche, Merck Serono, Sanofi, Celgene and AstraZeneca; Bayer consultant; Celgene consultant.

Alberto Sobrero: Advisory board participant for Roche, Bayer, Sanofi, Merck and Amgen.

Al Benson III: Research funding from Amgen, Genentech, Bayer, Novartis, Astellas and Gilead; scientific adviser to Bayer, Genentech, Genomic Health, Sanofi, Bristol-Myers Squibb and Lilly/Imclone.

Carmen Guillén Ponce: No conflicts of interest to declare.

Stefano Cascinu: Bayer consultant; Roche consultant; Novartis consultant; research funding from Merck Serono.

Eric Van Cutsem: Research funding from Bayer.

Iain R. Macpherson: Advisory board participant for Roche and Pierre-Fabre; travel support from Roche, Novartis and Celgene.

Dirk Strumberg: Bayer consultant.

Claus-Henning Köhne: Honoraria from Bayer; Bayer consultant.

John Zalcberg: Research funding and travel support from Bayer, Merck Serono and Roche; expert witness and member of speaker bureaux for Bayer.

Andrea Wagner: Employee of Bayer Pharma AG, Berlin, Germany.

Vittorio Luigi Garosi: Employee of Bayer S.p.A., Milan, Italy. 
Julia Grunert: Employee of Bayer Pharma AG, Wuppertal, Germany.

Josep Tabernero: Advisory role for Amgen, Bayer, Boehringer, Bristol-Myers Squibb, Genentech, Imclone, Lilly, Merck KGaA, Millennium, Novartis, Onyx, Pfizer, Roche and Sanofi.

Fortunato Ciardiello: Research funding from Merck Serono, Bayer and AstraZeneca; Merck Serono consultant; Bayer consultant.

\section{Acknowledgments}

The authors would like to thank the patients, their families and the study investigators. The CORDIAL trial was sponsored by Bayer HealthCare AG, Leverkusen, Germany. Writing support in the preparation of this manuscript was provided by Succinct Medical Communications. The authors retained full control over the content and the decision to publish the article.

\section{Appendix A. Supplementary data}

Supplementary data associated with this article can be found, in the online version, at http://dx.doi.org/10. 1016/j.ejca.2015.02.013.

\section{References}

[1] Globocan. Colorectal cancer. Estimated cancer incidence, mortality and prevalence worldwide in 2012. Available at: http://globocan. iarc.fr/Pages/fact_sheets_cancer.aspx; [last accessed 27.10.14].

[2] Van Cutsem E, Cervantes A, Nordlinger B, Arnold D. Metastatic colorectal cancer: ESMO Clinical Practice Guidelines for diagnosis, treatment and follow-up. Ann Oncol 2014;25(Suppl. 3):iii1-9.

[3] Yoo PS, Lopez-Soler RI, Longo WE, Cha CH. Liver resection for metastatic colorectal cancer in the age of neoadjuvant chemotherapy and bevacizumab. Clin Colorectal Cancer 2006;6:202-7.

[4] Benson III AB, Bekaii-Saab T, Chan E, Chen YJ, Choti MA, Cooper HS, et al. Metastatic colon cancer, version 3.2013: featured updates to the NCCN Guidelines. J Natl Compr Canc Netw 2013;11:141-52.

[5] Wilhelm SM, Dumas J, Adnane L, Lynch M, Carter CA, Schutz G, et al. Regorafenib (BAY 73-4506): a new oral multikinase inhibitor of angiogenic, stromal and oncogenic receptor tyrosine kinases with potent preclinical antitumor activity. Int J Cancer 2011;129:245-55.

[6] Grothey A, Van Cutsem E, Sobrero A, Siena S, Falcone A, Ychou M, et al. Regorafenib monotherapy for previously treated metastatic colorectal cancer (CORRECT): an international, multicentre, randomised, placebo-controlled, phase 3 trial. Lancet 2013;381:303-12.
[7] Schultheis B, Folprecht G, Kuhlmann J, Ehrenberg R, Hacker $\mathrm{UT}$, Kohne $\mathrm{CH}$, et al. Regorafenib in combination with FOLFOX or FOLFIRI as first- or second-line treatment of colorectal cancer: results of a multicenter, phase Ib study. Ann Oncol 2013;24:1560-7.

[8] Eisenhauer EA, Therasse P, Bogaerts J, Schwartz LH, Sargent D, Ford R, et al. New response evaluation criteria in solid tumours: revised RECIST guideline (version 1.1). Eur J Cancer 2009;45: $228-47$.

[9] Tournigand C, Andre T, Achille E, Lledo G, Flesh M, MeryMignard D, et al. FOLFIRI followed by FOLFOX6 or the reverse sequence in advanced colorectal cancer: a randomized GERCOR study. J Clin Oncol 2004;22:229-37.

[10] Carrato A, Swieboda-Sadlej A, Staszewska-Skurczynska M, Lim R, Roman L, Shparyk Y, et al. Fluorouracil, leucovorin, and irinotecan plus either sunitinib or placebo in metastatic colorectal cancer: a randomized, phase III trial. J Clin Oncol 2013;31: 1341-7.

[11] Hecht JR, Trarbach T, Hainsworth JD, Major P, Jager E, Wolff RA, et al. Randomized, placebo-controlled, phase III study of first-line oxaliplatin-based chemotherapy plus PTK787/ZK 222584, an oral vascular endothelial growth factor receptor inhibitor, in patients with metastatic colorectal adenocarcinoma. J Clin Oncol 2011;29:1997-2003.

[12] Saltz LB, Clarke S, Diaz-Rubio E, Scheithauer W, Figer A, Wong $\mathrm{R}$, et al. Bevacizumab in combination with oxaliplatin-based chemotherapy as first-line therapy in metastatic colorectal cancer: a randomized phase III study. J Clin Oncol 2008;26:2013-9.

[13] Hurwitz H, Fehrenbacher L, Novotny W, Cartwright T, Hainsworth J, Heim W, et al. Bevacizumab plus irinotecan, fluorouracil, and leucovorin for metastatic colorectal cancer. N Engl J Med 2004;350:2335-42.

[14] Bokemeyer C, Bondarenko I, Makhson A, Hartmann JT, Aparicio J, de Braud F, et al. Fluorouracil, leucovorin, and oxaliplatin with and without cetuximab in the first-line treatment of metastatic colorectal cancer. J Clin Oncol 2009;27:663-71.

[15] Van Cutsem E, Kohne CH, Hitre E, Zaluski J, Chang Chien CR, Makhson A, et al. Cetuximab and chemotherapy as initial treatment for metastatic colorectal cancer. N Engl J Med 2009;360:1408-17.

[16] Douillard JY, Siena S, Cassidy J, Tabernero J, Burkes R, Barugel $\mathrm{M}$, et al. Randomized, phase III trial of panitumumab with infusional fluorouracil, leucovorin, and oxaliplatin (FOLFOX4) versus FOLFOX4 alone as first-line treatment in patients with previously untreated metastatic colorectal cancer: the PRIME study. J Clin Oncol 2010;28:4697-705.

[17] Schmoll HJ, Cunningham D, Sobrero A, Karapetis CS, Rougier $\mathrm{P}$, Koski SL, et al. Cediranib with mFOLFOX6 versus bevacizumab with mFOLFOX6 as first-line treatment for patients with advanced colorectal cancer: a double-blind, randomized phase III study (HORIZON III). J Clin Oncol 2012;30:3588-95.

[18] Hoh C, Infante JR, Burris HA, Tarazi JC, Kim S, Rosbrook B, et al. Axitinib inhibition of [18F] fluorothymidine (FLT) uptake in patients (pts) with colorectal cancer (CRC): implications for cytotoxic chemotherapy combinations. J Clin Oncol 2011;29(Suppl.):3591. 